\title{
An Association of Vitamin D Insufficiency with Elevated Serum Uric Acid Levels Among Postmenopausal Women
}

${ }^{1}$ Mariya Ali, ${ }^{2}$ Tahira Naseem, ${ }^{3}$ Farooq Abdul Hakim Bajwa, ${ }^{4}$ Saira Zafar, ${ }^{4}$ Asma Akram, ${ }^{4}$ Hirra Ghaffar ${ }^{I}$ Department of Chemical Pathology, Continental Medical College, Lahore

${ }^{2}$ Department of Biochemistry \& Chemical Pathology, Shaikh Zayed Medical Complex, Lahore

${ }^{3}$ Department of Radiology, Lahore General Hospital, Lahore

${ }^{4}$ Department of Microbiology, Continental Medical College, Lahore

\begin{abstract}
Introduction: Deficiency of vitamin D and raised levels of serum uric acid are considered independent risk factors for causing cardiovascular diseases. Aims \& Objectives: This study was carried out to study Serum Uric acid and Vitamin D levels in postmeopausal women to find association of hypovitaminosis D with hyperuricemia. Place and duration of study: It was a cross-sectional analytical study. Postmenopausal women more than 55 years of age with BMI between $25-30 \mathrm{~kg} / \mathrm{m}^{2}$ were included in the study. The study was conducted at the Department of Biochemistry and Chemical Pathology, Shaikh Zayed Federal Postgraduate Medical Institute, Lahore over a period of six months. Material \& Methods: Eighty subjects were included in this study. They were divided into two groups i-e, normouricemic group and hyperuricemic group based onserum uric acid level.Vitamin D levels of both groups were then measured. Results: The hyperuricemic group had markedly lower vitamin Dlevels as compared to normouricemic group. Moreover, vitamin D had a negative correlation with uric acid in the hyperuricemic group i-e, higher the uric acid level, lower was vitamin D. Multiple regression analysis was also performed for studying the relation of vitamin D with uric acid levels in the two groups overall. It was observed that one $\mathrm{mg} / \mathrm{dl}$ increase in uric acid caused an average decrease of $2.43 \mathrm{ng} / \mathrm{ml}$ in vitamin D. Conclusion: It may be concluded from this study that association of hypovitaminosis D with hyperuricemia in postmenopausal women can be used for planning an early intervention to prevent cardiovascular diseases in them.
\end{abstract}

Key words: Hyperuricemia, Hypovitaminosis D, Cardiovascular diseases

\section{INTRODUCTION}

$\mathrm{H}$ yperuricemia is considered to be a predictor for cardiovascular diseases ${ }^{1}$ and at the same time deficiency of vitamin $\mathrm{D}$ is also considered an independent risk factor for the development of cardiovascular diseases. ${ }^{2}$ Hypovitamnosis D and hyperuricemia are being considered major public health concerns worldwide and the prevalence has been increasing in both developed and developing countries. $^{3}$

Vitamin D deficiency is defined as the serum level of 25-hydroxy vitamin D less than $20 \mathrm{ng} / \mathrm{ml}$ while a serum level of 25 hydroxy vitamin $\mathrm{D}$ in the range $20-29.9 \mathrm{ng} / \mathrm{ml}$ falls into the category of vitamin D insufficiency. ${ }^{4}$ Approximately 1 billion people throughout the world are suffering from vitamin D deficiency. ${ }^{5}$ A study conducted in Pakistan on about
200 postmenopausal women showed that $59 \%$ of these women were vitamin D deficient. ${ }^{6}$ It has been documented that risk of myocardial infraction doubles in patients having vitamin D levels $<34 \mathrm{ng} / \mathrm{ml}^{7}$ Studies show that serum vitamin D of more than $30 \mathrm{ng} / \mathrm{ml}$ is implicated in preventing cardiovascular diseases. ${ }^{8}$

Uric acid (2,6,8-trihydroxypurine) is a naturally occurring nitrogenous waste product in the body. ${ }^{9}$ The reference interval in plasma or serum for females is $2.6-6.0 \mathrm{mg} / \mathrm{dl}$ and in the twenty four hour urine of adult it is $250-750 \mathrm{mg} / \mathrm{dl} .{ }^{10}$ Hyperuricemia is a metabolic disorder characterized by either raised uric acid synthesis or decreased renal excretion. ${ }^{11}$ Women are considered to have hyperuricemia if their serum uric acid level is $\geq 6.0 \mathrm{mg} / \mathrm{dl} .{ }^{15} \mathrm{In}$ Pakistan about $27 \%$ of adult females have been found to be hyperuricemic. ${ }^{12}$ Hyperuricemia is found to be more prevalent in postmenopausal 
women, some ethnic groups like African Americans and patients with renal diseases. ${ }^{13}$ Raised serum uric acid (SUA) in postmenopausal women is linked to the lack of estrogen which is believed to be involved in the excretion of uric acid. ${ }^{14}$

Excess uric acid has been related to the risk of hypertension, atherosclerosis and cardiovascular conditions. ${ }^{15}$ Relationship of raised SUA level with vitamin $\mathrm{D}$ insufficiency is a significant metabolic disorder that is associated with cardiovascular diseases. ${ }^{16}$ Low levels of vitamin D cause the release of parathyroid hormone through the activation of parathyroid gland. ${ }^{17}$ Some previous studies showed that high levels of parathyroid hormone increase the occurrence of hyperuricemia. ${ }^{18}$ It is believed that there are some absorptive and secretory transporters in renal tissue at the basolateral and apical membranes which are involved in the transportation of uric acid. ${ }^{19}$ Parathyroid hormone affects some of the transporters of proximal tubules of kidney, for example, its inhibition of sodium/hydrogen ion exchange type 3 isoform is known. ${ }^{20}$ Renal secretion of uric acid is reduced among hyperuricemic patients having high levels of parathyroid hormone, the severity of which relies on the change in the rate of absorption. ${ }^{21}$

The current study has been designed to study serum uric acid and vitamin D levels and to find out the relationship of low levels of vitamin $\mathrm{D}$ with high serum uric acid levels among postmenopausal women in our population. Due to an increasing trend of hyperuricemia and hypovitaminosis D in the middle aged and elderly population, the present study might have significant public health implications and it may also help out in planning an appropriate intervention to prevent cardiac diseases in these patients. Improved exposure to the sun, better diet, supplementation of vitamin $\mathrm{D}$ and measures to reduce serum uric acid may therefore be utilized to improve general health, normalize serum uric acid and prevent cardiovascular diseases.

\section{MATERIAL AND METHODS}

It was a "cross-sectional analytical study". The samples were collected from a medical camp in Bajwa Clinic, Kahna, Ferozpure Road, Lahore. Sample size was 80 which was estimated for each group by using $95 \%$ confidence level. $90 \%$ power of test with expected mean vitamin D levels of $43 \pm 12$ and $35 \pm 10$ in uric acid normal and uric acid elevated group respectively. ${ }^{22}$ This was calculated by using power and precision 3.0 software. The sampling technique was non probability convenient sampling.
Postmenopausal women above 55 years of age and having BMI between 25-30 were included in the study. Patients with self-reported gout or arthritis, self-stated parathyroid or thyroid disease, having used uric acid lowering medicine in last 14 days, deranged liver function tests, history of chronic renal disease, tumours and cardiac disease were excluded from the study. After approval from Institutional Review Board of FPGMI (letter no.F38/NHRC/ADMIN/IRB/226), consent forms were filled and signed by the participants of study. Serum Uric acid assay was done by uricase method on fully automated chemistry autoanalyzer AU480 by Beckmann Coulter and subjects were divided into two groups based on their uric acid level i.e, normouricemic and hyperuricemic. Each group comprised of 40 postmenopausal women. Vitamin $\mathrm{D}$ levels of both groups were then measured by chemiluminescent immunoassay on fully automated analyzer Access 2 by Beckman Coulter.

\section{Statistical analysis:}

Data analysis was performed using latest version of SPSS 22.0. Mean \pm SD and median of the two parameters was obtained. Statistical significance was calculated by Student's t-test and Mann Whitney U Test. $\mathrm{P}<0.05$ was considered significant. Step wise regression analysis was done to determine the effect of SUA on Vitamin D.

\section{RESULTS}

The study included 80 postmenopausal women, 40 having normal uric acid level and 40 hyperuricemic. The mean age for normouricemic group was $59.4 \pm 4.4$ years and that of hyperuricemic group was $61.1 \pm 3.5$ years. On comparing the vitamin D levels of both groups it was found that hyperuricemic group had relatively close range of vitamin D levels while for normouricemic the range was quite wide with a significant $p$-value of 0.006 . In normouricemic group there were 12 cases of vitamin D deficiency while 19 cases were of vitamin $\mathrm{D}$ insufficiency. On the other hand in hyperuricemic group there were 19 cases of vitamin D deficiency and 19 cases fell into vitamin D insufficiency. pvalue of 0.049 was significant on comparison of both groups. 


\begin{tabular}{|c|c|c|c|}
\hline & \multicolumn{2}{|c|}{ Group } \\
\hline & & Normouricemic & Hyperuricemic \\
\hline \multirow{5}{*}{$\begin{array}{l}\text { Vitamin D } \\
(\mathrm{ng} / \mathrm{ml})\end{array}$} & Mean & 24.9 & 19.5 \\
\hline & $\mathrm{SD}$ & 12.3 & 5.0 \\
\hline & $\mathrm{Q}_{1}$ & 18.2 & 17.3 \\
\hline & Median & 23.7 & 20.1 \\
\hline & $\mathrm{Q}_{3}$ & 28.2 & 22.2 \\
\hline \multicolumn{2}{|c|}{ Mann Whitney $=516.5$} & \multicolumn{2}{|c|}{ P-value $*=0.006$} \\
\hline \multirow{3}{*}{$\begin{array}{l}\text { Vitamin } \\
\text { D status }\end{array}$} & $\begin{array}{c}<20.0 \\
\text { (deficient) }\end{array}$ & 12 & 19 \\
\hline & \begin{tabular}{|c|}
$20.0-29.9$ \\
(insufficient)
\end{tabular} & 19 & 19 \\
\hline & $\begin{array}{c}30.0+ \\
\text { (sufficient) }\end{array}$ & 9 & 2 \\
\hline
\end{tabular}

Chi-square $=6.04$

Table-1: Vitamin D levels of study subjects of both groups based on SUA level.

$\mathrm{P} *$ means that $\mathrm{p}$ value is $<0.05$ (significant)

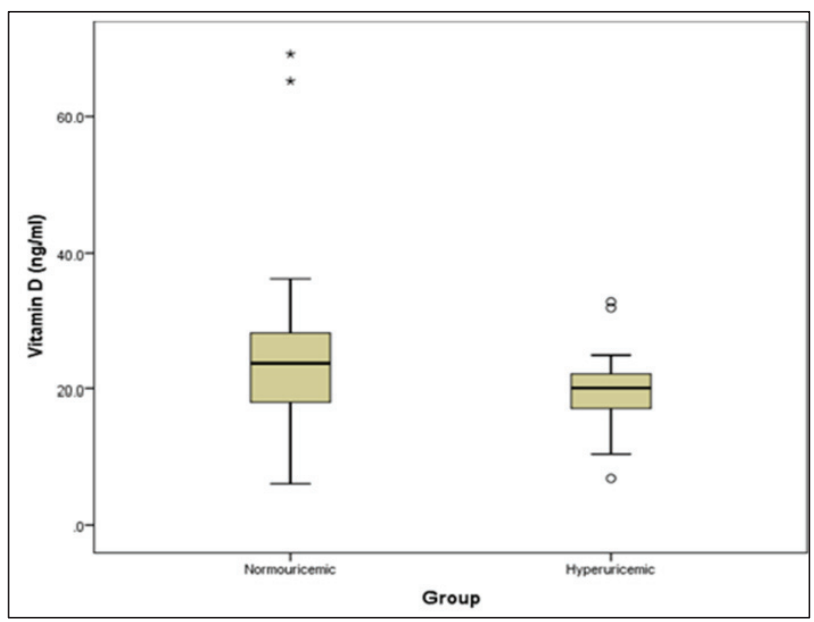

Fig-1: Boxplot presenting distribution for vitamin D levels amongst both group of study subjects.

The correlation analysis was performed to see the association of vitamin $\mathrm{D}$ with uric acid. This analysis was carried out within each group and overall. It was noted that overall uric acid had significant negative correlation with vitamin $\mathrm{D}$ with $\mathrm{r}=-0.471$ with $\mathrm{p}$-values $<0.001$. When the correlation analysis was performed in hyperuricemic group it was noted that the uric acid level had a good negative correlation of -0.577 with vitamin $\mathrm{D}$ showing that higher the uric acid levels lower the vitamin D levels.

The multiple linear regression analysis was done to see the effect of uric acid on vitamin D. Result found at $17^{\text {th }}$ step showed that the final significant model was decided. It was noted that the uric acid had a significant impact on vitamin D level. When all 80 cases were included, the average vitamin D level was $35.53 \mathrm{ng} / \mathrm{ml}$ and one unit increase in uric acid caused an average decrease of $2.43 \mathrm{ng} / \mathrm{ml}$ in vitamin D.

\begin{tabular}{|l|l|c|c|c|c|}
\hline \multicolumn{2}{|l|}{} & \multicolumn{2}{|c|}{$\begin{array}{l}\text { Unstandardized } \\
\text { Coefficients }\end{array}$} & \multirow{2}{*}{$T$} & P-value \\
\cline { 3 - 4 } & B & Std. Error & & \\
\hline \multirow{2}{*}{17} & \begin{tabular}{l} 
(Constant) \\
\cline { 2 - 3 }
\end{tabular} & 35.53 & 3.52 & 10.11 & $0.000^{*}$ \\
\cline { 2 - 5 } & $\begin{array}{l}\text { Uric Acid } \\
(\mathrm{mg} / \mathrm{dl})\end{array}$ & -2.43 & 0.61 & -3.94 & $0.000^{*}$ \\
\hline
\end{tabular}

Table-2: Multiple linear regression analysis for studying relation of vitamin $\mathrm{D}$ with uric acid by using Backward method in both groups combined (R2 $=0.166$ Adj R2=0.156)

Dependent Variable: Vitamin D (ng/ml)

The same model was applied on hyperuricemic group and it was noted that in this group serum uric acid had highly significant and negative effect on vitamin $\mathrm{D}$ with $\mathrm{p}$-values $<0.050$. This model was obtained in $12^{\text {th }}$ step with adjusted $\mathrm{R}^{2} 0.555$ showing reasonably good fit model. An increase of one $\mathrm{mg} / \mathrm{dl}$ in uric acid level in this group caused an average decrease of $2.02 \mathrm{ng} / \mathrm{ml}$ in vitamin $\mathrm{D}$.

\section{DISCUSSION}

Eighty postmenopausal women were included in this study. They were divided into two groups: normouricemic and hyperuricemic groups. The difference between the ages of hyperuricemic and normouricemic groups was insignificant. On comparing vitamin D levels of both groups it came into notice that mean vitamin $\mathrm{D}$ level for hyperuricemic group was lower as compared to normouricemic subjects. The difference was significant with p-value of 0.006.The results are similar to a study by Takir $M$ et al in which hyperuricemic group had lower values of vitamin D as compared to normouricemic subjects. There was a negative and important relationship between SUA and vitamin D. ${ }^{23}$ Similar findings were also seen in another study done in Turkey. In this research vitamin $\mathrm{D}$ and uric acid levels of the patients were obtained. Their findings suggested that subjects having increased SUA levels had low levels of vitamin $\mathrm{D}$. They concluded that low level of vitamin D were correlated with high serum uric acid levels. ${ }^{24}$ Correlation analysis was performed to see the relationship of vitamin $\mathrm{D}$ with uric acid within each group and overall. When multiple regression analysis was performed it was found that uric acid had a significant impact on vitamin $\mathrm{D}$. These results are in agreement with a study done in China in which multiple regression analysis was performed and it was found that there was an independent and significant relationship between vitamin D insufficiency and high SUA levels among the postmenopausal women. The subjects with vitamin 
D insufficiency were more likely to have raised SUA compared with those without vitamin D insufficiency among postmenopausal women. Their findings suggested that vitamin $\mathrm{D}$ status is negatively associated with elevated serum uric acid. They also summarized that exposure to the sunlight and vitamin D supplementation are easy as well as an inexpensive ways for preventing low levels of vitamin $\mathrm{D}$ and other health issues. ${ }^{22}$ Another study conducted in Saudi Arabia concluded that vitamin D was inversely related with serum uric acid level. ${ }^{25} \mathrm{~A}$ study from Egypt also concluded that low level of vitamin D was inversely correlated with high serum uric acid level. ${ }^{26}$ A study carried out in Turkey suggested that patients who have elevated serum uric acid had lower vitamin D levels. ${ }^{27}$ Another research carried out on 100 patients for finding out the possible effect of vitamin D on uric acid level concluded that there was an inverse relationship between vitamin $\mathrm{D}$ and serum uric acid. ${ }^{28}$

A systemic review was conducted in 2019 which showed that patients with vitamin D deficiency and insufficiency had a significantly higher level of serum uric acid compared with individuals with normal vitamin D. ${ }^{29}$

\section{CONCLUSION}

It may be concluded from our study that postmenopausal women with higher serum uric acid level had lower level of vitamin D as compared to those who had normal serum uric acid levels. A negative correlation was observed between serum uric acid and vitamin D level in the group with hyperuricemia. Low vitamin $\mathrm{D}$ and raised serum uric acid are important risk factors for the development of cardiovascular diseases. By improving vitamin D level, serum uric acid can be normalized. Association of hypovitaminosis D with hyperuricemia in postmenopausal women can be used in planning an early intervention to prevent cardiac diseases in such subjects.

Limitations of study: It was a single center study with a small sample size which may not be sufficient to correlate low levels of vitamin D with elevated SUA. A study should be carried out with larger sample size and in different socio economic strata to get more accurate information.

\section{REFERENCES}

1. Borghi C, Cicero AF. Serum uric acid and acute coronary syndrome: Is there a role for functional markers of residual cardiovascular risk? Int. J. Cardiol. 2018; 250:62-3.
2. Kim SH, Oh JE, Song DW, Cho CY, Hong SH, Cho YJ, Yoo BW, Shin KS, Joe H, Shin HS, Son DY. The factors associated with Vitamin D deficiency in community dwelling elderly in Korea. Nutr Res Pract. 2018; 12(5):387-95.

3. Liu H, Zhang X M, Wang Y. L, Liu B-C. Prevalence of hyperuricemia among Chinese adults: a national cross-sectional survey using multistage, stratified sampling. JNephrol. 2014; 27(6):653-8.

4. Pfotenhauer KM, Shubrook JH. Vitamin D deficiency, its role in health and disease, and current supplementation recommendations. J Am Osteopath Assoc. 2017; 117(5):301-5.

5. Ilson LR, Tripkovic L, Hart KH, Lanham-New SA. Vitamin D deficiency as a public health issue: using vitamin D 2 or vitamin D 3 in future fortification strategies. Brit J Nutr. 2017; 76(3):392-9.

6. Akhtar S, Jan R. Prevalence of Vitamin D Inadequacy among Postmenopausal Women. Med Forum Mon J Spec. 2019; 24(11):58-9.

7. Kheiri B, Abdalla A, Osman M, Ahmed S, Hassan M, Bachuwa G. Vitamin D deficiency and risk of cardiovascular diseases: a narrative review. J. Clin. Hypertens. 2018; 24(1):1-9.

8. Mozos I, Marginea O. Links between vitamin D deficiency and cardiovascular diseases. Biomed Res. Intern. 2015; (12).

9. Hafez RM, Abdel-Rahman TM, Naguib RM. Uric acid in plants and microorganisms: Biological applications and genetics-A review. J. Adv. Res. 2017; 8(5):475-86.

10. Maiuolo J, Oppedisano F, Gratteri S, Muscoli C, Mollace V. Regulation of uric acid metabolism and excretion. Int. J. Cardiol. 2016; 213:8-14.

11. Yokokawa H, Fukuda H, Suzuki A, Fujibayashi $\mathrm{K}$, Naito $\mathrm{T}$, Ueharo $\mathrm{A}$, et al. Association between serum uric acid levels /hyperuricemia and hypertension among 85, 286 Japenese workers. J Clin Hypertens. 2016; 18(1):53-9.

12. Raja S, Kumar A, Ahooja R, et al. Frequency of hyperuricemia and its risk factors in Adult population. Cureus. 2019; 11(3):4198-4202.

13. Cho SK, Winkler CA, Lee SJ, Chang Y, Ryu S. The Prevalence of Hyperuricemia Sharply Increases from the Late Menopausal Transition Stage in Middle-Aged Women. J. Clin. Med. 2019; 8(3):296.

14. Naseem R, Zafar SM, Jawed S, Mukhtar S, Ijaz $\mathrm{F}$, Aftab RK. Influence of serum estradiol on serum uric acid level in pre and postmenopausal women. Prof. Med. J. 2019; 26(09):1587-91. 
15. Muiesan ML, Agabiti-Rosei C, Paini A, Salvetti M. Uric acid and cardiovascular disease: an update. Eur Cardiol. 2016; 11(1):54.

16. Thakkinstian A, Anothaisintawee T, Chailurkit L, Ratanachaiwong W, Yamwong S, Sritara P, Ongphiphadhanakul B. Potential causal associations between vitamin $\mathrm{D}$ and uric acid: Bidirectional mediation analysis. Scientific reports. 2015; 5:14528.

17. Martins JS, Palhares MD, Teixeira OC, Gontijo Ramos M. Vitamin D status and its association with parathyroid hormone concentration in Brazilians. Nutr Metab. Jan 12017.

18. Sugimoto R, Watanabe H, Ikegami K, Enoki Y, Imafuku T, Sakaguchi Y, Murata M, Nishida K, Miyamura S, Ishima Y, Tanaka M. Downregulation of $\mathrm{ABCG} 2$, a urate exporter, by parathyroid hormone enhances urate accumulation in secondary hyperparathyroidism. Kidney Int. 2017; 91(3):658-70.

19. Girardi C, Titan S, Malnic G, Reboucas N. Chronic effect of parathyroid hormone on NHE3 expression in renal proximal tubules. Kidney Int .2000; 58(4):1623-31.

20. Hisatome I, Ishimura M, Sasaki N. Renal handling of urate in two patients with hyperuricemia and primary hyperparathyroidism. Int Med J. 1992; 31(6):807-11.

21. Wen C, David C, Chan H, Tasai M, Chung W. Is a high serum uric acid a risk marker or target for treatment. Am J Nephrol. 2010;56(2):273-88

22. Peng $\mathrm{H}$, Li $\mathrm{H}$, Li $\mathrm{C}$, Chao $\mathrm{X}$, Zhang $\mathrm{Y}$. Association between Vitamin D Insufficiency and Elevated serum uric acid among middle aged and elderly Chinese Han Women. Pubmed. 2013; 8(4):1-9.

23. Takir M, Solak Y, Erek A, Kostek O, Oral A, Elcioglu o, et al. Association between serum uric acid and vitamin D insufficiency among middle aged and elderly population. Turk Neph Dial Transpl. 2016; 25(2):182-6.

24. Takir M, Tprok E, Elcioglu C, Bakan A, Kostek A, Cakhh T, et al. Is there a link between vitamin D and uric acid?. Clin Chem Lab Med. 2014; 52(1):22-6.

25. Gehan et al. Relationship between hyoeruricemia and vitamin D deficiency among adults in Al Kharj, Saudia Arabia. Intr J Adv Res. 2015; 3(1):269-273.

26. Khalid Al Syed, Magdy Elasyed, Reem Sabry. Relationship between serum uric acid and vitamin D among elderly Egyptians. J Am Sci. 2014; 9(12):984-988.
27. Yandi Y, Geenra F, Kilauz B, Bakin A, Sahim $\mathrm{H}$, Billi $\mathrm{M}$ et al. Cyanocobalamin and 25 hydroxy vitamin D levels in gout; an overlooked issue. OJRA An Acadm Publsh.2016:6(4):96-101

28. Aakay E, Korakaelci F, Gurosay G, Karabag Y, Yilidiz M, Erodgu H, et al .The Possible effect of vitamin D on Uric Acid in Diabetic patients. World Clin J Med Sci. 2017; 1(2):77-83.

29. Charoenngam N, Ponvilawan B, Ungprasert P. Vitamin D insufficiency and deficiency are associated with a higher level of serum uric acid: A systematic review and meta-analysis. Modern rheumatology. 2019 Mar4.

\section{The Authors:}

Dr. Mariya Ali,

Demonstrator,

Department of Chemical Pathology,

Continental Medical College, Lahore.

Dr. Tahira Naseem

HOD, Biochemistry and Chemical Pathology,

Shaikh Zayed Medical Complex, Lahore.

Dr. Farooq Abdul Hakim Bajwa,

Medical Officer,

Department of Radiology,

Lahore General Hospital, Lahore.

Dr. Saira Zafar,

Assistant Professor,

Department of Microbiology,

Continental Medical College, Lahore.

Dr. Asma Akram,

Demonstrator,

Department of Pathology,

Continental Medical College, Lahore.

Hirra Ghaffar

Demonstrator,

Department of Pathology,

Continental Medical College, Lahore.

\section{Corresponding Author:}

Dr. Mariya Ali,

Demonstrator,

Department of Chemical Pathology,

Continental Medical College, Lahore.

E-mail: drmariafarooq@hotmail.com 